\title{
The Association between Serum Estradiol and Progesterone on the Same Day of FET and the Pregnancy Outcome, a cross-sectional study
}

\author{
Kamal Rageh ${ }^{1}$, Ahmed Barakat ${ }^{2}$, and Nada Barakat ${ }^{2}$ \\ ${ }^{1}$ Al-Azhar University \\ ${ }^{2}$ Al-Baraka fertility hospital
}

October 8, 2020

\begin{abstract}
Research question: Precise timed synchronization between endometrium and the embryo is essential for high implantation and pregnancy rate, it is worthy to mention that endometrial thickness is not the only factor, E2 and P levels are also regularly monitored for endometrial receptivity. So, we decided to go for this study, to investigate the impact of serum E2 and P levels on the same day of embryo transfer on pregnancy outcomes for FET cycles. Design: This was a retrospective cross sectional study for 402 FET cycles which conducted between April 2018 and May 2019. All participants started endometrial preparation for FET with $6 \mathrm{mg} /$ day oral estradiol for 13 days. When endometrium reached $8 \mathrm{~mm}$ or greater, patients were initiated on both micronized vaginal and oral $\mathrm{P}$ treatment. On FET day, serum level of E2 and P were assessed. Then, transfer of PGT euploid embryos was performed. 12 days later pregnancy test was assessed, and then 4 weeks after FET date ultrasound was scheduled to check the viability and the clinical pregnancy. Results: The mean E2 value was $931.41 \pm 438.65 \mathrm{pg} / \mathrm{ml}, \mathrm{while}$ mean $\mathrm{P}$ value was $8.47 \pm 9.4 \mathrm{ng} / \mathrm{ml}$. 240 out of 402 cases got pregnant $(59.7 \%)$ while the clinical pregnancy rate was $53.9 \%$ with no correlation between serum (E2, P \& E/P ratio) and the outcome. Conclusion: Our results revealed that the association between E2 and P on FET day and the pregnancy outcome is still not proven and those markers can't serve as predictors for the outcome.
\end{abstract}

Research question:

Precise timed synchronization between endometrium and the embryo is essential for high implantation and pregnancy rate, it is worthy to mention that endometrial thickness is not the only factor, E2 and P levels are also regularly monitored for endometrial receptivity. So, we decided to go for this study, to investigate the impact of serum E2 and P levels on the same day of embryo transfer on pregnancy outcomes for FET cycles.

Design:

This was a retrospective cross sectional study for 402 FET cycles which conducted between April 2018 and May 2019. All participants started endometrial preparation for FET with $6 \mathrm{mg} /$ day oral estradiol for 13 days. When endometrium reached $8 \mathrm{~mm}$ or greater, patients were initiated on both micronized vaginal and oral $\mathrm{P}$ treatment. On FET day, serum level of E2 and P were assessed. Then, transfer of PGT euploid embryos was performed. 12 days later pregnancy test was assessed, and then 4 weeks after FET date ultrasound was scheduled to check the viability and the clinical pregnancy.

Results:

The mean E2 value was $931.41 \pm 438.65 \mathrm{pg} / \mathrm{ml}$, while mean $\mathrm{P}$ value was $8.47 \pm 9.4 \mathrm{ng} / \mathrm{ml}$. 240 out of 402 
cases got pregnant $(59.7 \%)$ while the clinical pregnancy rate was $53.9 \%$ with no correlation between serum (E2, $\mathrm{P} \& \mathrm{E} / \mathrm{P}$ ratio) and the outcome.

Conclusion:

Our results revealed that the association between E2 and P on FET day and the pregnancy outcome is still not proven and those markers can't serve as predictors for the outcome.

\section{Hosted file}

NEW FET paper 2020.pdf available at https://authorea.com/users/365458/articles/485559-theassociation-between-serum-estradiol-and-progesterone-on-the-same-day-of-fet-and-thepregnancy-outcome-a-cross-sectional-study 